\title{
Applications of capillary electrophoresis in DNA mutation analysis of genetic disorders
}

\author{
H Le, D Fung, R J Trent
}

\begin{abstract}
Aim-To facilitate DNA mutation analysis by use of capillary electrophoresis.

Methods-The usefulness and applications of capillary electrophoresis in DNA fragment sizing and sequencing were evaluated.

Results-DNA mutation testing in disorders such as cystic fibrosis, Huntington disease, $\alpha$ thalassaemia, and hereditary fructose intolerance were undertaken effectively. However, sizing the (CAG) $n$ repeat in the case of Huntington disease was a potential problem when using capillary electrophoresis. Separation polymers used in capillary electrophoresis are still in the developmental phase, with improved ones being released regularly. Conclusions-In the DNA diagnostic setting, capillary electrophoresis is a valuable development because it expands the scope for automation and has useful analytical properties. The potential to perform complex multiplexing within one electrophoresis run facilitates DNA diagnosis. The different mobility of DNA fragments in capillary electrophoresis compared with conventional gel electrophoresis will require, in some circumstances, additional care when results are being interpreted or reported. Capillary electrophoresis is a cheap alternative for combined automated sequencing and fragment analysis that utilises multicolour fluorescence capability. However, in its present form, it is not useful for large scale sequencing.

(F Clin Pathol: Mol Pathol 1997;50:261-265)
\end{abstract}

Keywords: capillary electrophoresis; DNA mutation analysis; automation

Microsatellite analysis, scanning for DNA mutations, DNA sequencing, and other approaches used for the detection of mutations require a common and rate limiting step that involves the use of slab gel electrophoresis to separate DNA fragments. ${ }^{1}$ Slab gels, whether they are made from agarose or polyacrylamide, take time to prepare and run. They can be a source of error in the form of band shifts or "smiling". DNA fragments in slab gels need to be visualised by staining or autoradiography following which an assessment of the band patterns enables a genetic diagnosis to be made. This assessment requires sizing of fragments or, in the case of DNA sequencing, an ordering of fragments relative to each other. As well as being time consuming, frequently the visualisation step involves the use of toxic substances, such as ethidium bromide or radioactive labels. Also, errors can occur because gels are read manually.

An alternative to slab gel electrophoresis is capillary electrophoresis. In the latter, DNA fragments are separated into their respective sizes following migration in an electric field produced within a capillary. ${ }^{2}$ A very high voltage $(\sim 15 \mathrm{kV})$ can be used to generate the electrical field because heat is dissipated easily in a capillary. Movement of molecules in capillary electrophoresis is complex, as it is dependent not only on their mass and net charge but can also be affected by the electro-osmotic flow. ${ }^{2}$ This occurs because in capillary electrophoresis, the conducting solution can also move under the influence of the electric field. Capillaries are made of fused silica, the inner surface of which becomes negatively charged following contact with the conducting buffer, particularly at a high $\mathrm{pH}$. To maintain electroneutrality, cations build up on this surface. Following application of an electrical field, these cations migrate towards the cathode, taking water molecules with them. The effect of this is a net flow of solution along the capillary. ${ }^{2}$ Under the influence of the electro-osmotic flow, cations will migrate towards the cathode faster, followed by neutral molecules, and lastly anions. The electro-osmotic effect can be used to separate analytes more effectively or it can be inhibited by coating the capillaries. The high voltage, as well as the effect of electro-osmotic flow, enhance the resolution of DNA fragments.

The use of a replaceable liquid polymer abolishes the need for gel casting that, in association with automated sample loading and data analyses, reduce the potential for human error. The scope for multiplexing is increased if the instrument allows a number of different coloured fluorochromes to be detected simultaneously. In this study, we describe our experience with capillary electrophoresis in a routine DNA diagnostic service. We also illustrate the usefulness and problems of this technique with reference to several genetic disorders including cystic fibrosis, Huntington disease, $\alpha$ thalassaemia, hereditary fructose intolerance, and Angelman syndrome.

\section{Methods}

DNA PREPARATION AND PROCESSING

Genomic DNA was isolated from blood leucocytes and chorionic villus samples using a standard phenol/chloroform method. For 
cystic fibrosis testing, DNA obtained by a rapid extraction method, involving the use of a lysis buffer and heating to $55^{\circ} \mathrm{C}$ for one hour, was adequate. For DNA amplification by polymerase chain reaction (PCR), one of the two primers was labelled at its $5^{\prime}$ end with the fluorochrome 6-FAM. The following mutations were sought on the basis of fragment size differences between mutant and wild-type alleles: 3 base pairs $\Delta F 508$ deletion in cystic fibrosis, the $(\mathrm{CAG})_{\mathrm{n}}$ triplet repeat in Huntington disease, and the $\sim 20 \mathrm{~kb}$ deletion in the South East Asian type $\alpha$ thalassaemia. One of the two primers for microsatellite AFM320 (D15S210) was labelled with the dye HEX. PCR for Huntington disease testing was also undertaken with a set of different primers and the addition of ${ }^{32} \mathrm{P}$ to the reaction mixture. Exon 5 of the aldolase B gene (360 base pairs) and exon 3 of the UBE3A gene (1246 base pairs) were amplified and sequenced using the ABI PRISM ${ }^{\mathrm{TM}}$ Dye Terminator Cycle Sequencing Ready Reaction kit. PCR conditions and primer sequences are available on request. Internal size markers labelled with the dye TAMRA (GS500, GS2500) were purchased from Perkin-Elmer (Foster City, California, USA). These cover size ranges 35-500 and 40-14 000 base pairs, respectively.

CAPILLARY ELECTROPHORESIS

This was performed with the ABI PRISMTM 310 Genetic Analyzer (Perkin-Elmer). Separation polymers in which DNA was electrophoresed included the Performance Optimized Polymer 4 that contains a denaturant, and is used at $60^{\circ} \mathrm{C}$ (fragment analysis) and the DNA sequencing polymer. These, as well as the $10 \times$ Gene Scan Analyzer buffer, with and without EDTA, and capillaries, were obtained from Perkin-Elmer. Ultra pure grade formamide was obtained from Amresco (Solon, Ohio, USA) and analytical grade dimethyl sulphoxide from Ajax Chemicals (Auburn, New South Wales, Australia). Formamide $(10 \mathrm{ml})$ was deionised overnight with $1 \mathrm{~g}$ Amberlite MB-1A mixed bed exchanger (Sigma, St Louis, Missouri, USA) and then frozen in $1.5 \mathrm{ml}$ aliquots.

\section{DNA fragment sizing}

A volume of $1 \mu \mathrm{l}$ of the PCR mixture (amplicon) was diluted with $12 \mu \mathrm{l}$ deionised formamide and $0.5 \mu \mathrm{l}$ of DNA size marker GS500 TAMRA or GS2500 TAMRA. When PCR products were present in excess, the amplicon was diluted one in five before the $1 \mu \mathrm{l}$ was taken. The PCR/formamide/DNA size marker mixture was vortexed briefly and heated to $95^{\circ} \mathrm{C}$ for four minutes and then placed on ice before being loaded on to the ABI PRISM ${ }^{\text {TM }} 310$ Genetic Analyzer's 48 slot autosampler (a 96 sample version is also available). The mixture was electrophoresed using a $47 \mathrm{~cm} / 50 \mu \mathrm{m}$ internal diameter uncoated capillary. The default settings for injection (loading) and running times were: injections, five seconds at $15 \mathrm{kV}$; electrophoresis, $15 \mathrm{kV}$ for 16 minutes at $60^{\circ} \mathrm{C}$. However, following optimisation studies, the injection conditions needed to be modified depending on the amplicon involved. The amount of sample loaded could be changed by injecting electrokinetically for periods of one to five seconds at $10 \mathrm{kV}$ or $15 \mathrm{kV}$. Electrophoresis in EDTA containing $1 \times$ GeneScan Analyzer buffer remained constant at $15 \mathrm{kV}$ and $60^{\circ} \mathrm{C}$, but the run varied from 음 12-30 minutes depending on the amplicon 0 size. Data were collected and analysed using $\stackrel{0}{5}$ the ABI PRISM ${ }^{\mathrm{TM}}$ Data Collection software and GENESCANTM Analysis software, respectively.

\section{DNA sequencing}

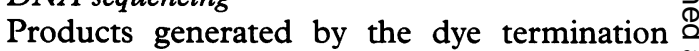
method were purified using Centri-Sep col- के umns (Princeton Separations, Princeton, New $\vec{\circ}$ Jersey, USA) and precipitated by adding $400 \mu \mathrm{l} \vec{\overrightarrow{ }}$ of ice cold $100 \%$ ethanol. The dried sequenc- $\vec{\omega}$ ing products were reconstituted in $25 \mu \mathrm{l}$ of $\frac{\Omega}{3}$ Template Suppressor Reagent and heated at $95^{\circ} \mathrm{C}$ for four minutes before being separated $\delta$ in the ABI PRISM ${ }^{\text {TM }} 310$ Genetic Analyzer ir equipped with an internally coated $47 \mathrm{~cm} / \stackrel{\text { S }}{\rightarrow}$ $7.5 \mu \mathrm{m}$ internal diameter capillary containing 응 the DNA sequencing polymer. The products were injected electrokinetically at $2.5 \mathrm{kV}$ for $30 \overrightarrow{\mathrm{O}}$ seconds and electrophoresed in $1 \times$ GeneScan $\frac{9}{0}$ Analyzer buffer without EDTA at $6 \mathrm{kV}$ for 120 minutes at $42^{\circ} \mathrm{C}$. Sequence data were edited with the ABI PRISM ${ }^{\text {TM }}$ DNA Sequencing Analysis software.

\section{Results}

CYSTIC FIBROSIS

When testing for the $\Delta \mathrm{F} 508$ mutation, mutant (91 base pairs) and wild-type (94 base pairs) alleles were distinguished easily. The alleles $\overrightarrow{\overrightarrow{0}}$ showed the expected three base pair difference (deletion of a codon for phenylalanine) but were smaller than would be expected from DNA sequence data (95 and 98 base pairs, respectively). The same DNA fragments analysed by conventional slab gel electrophoresis would be difficult to size accurately. However, 8 they are usually designated as being 95 and 98 base pairs on the basis of an approximation 을 obtained by visual comparison with DNA size markers. Despite the fragment sizing difficulty, the excellent reproducibility obtainable with $\bar{N}$ the ABI PRISM ${ }^{\text {TM }} 310$ Genetic Analyzer was illustrated by cystic fibrosis testing. In $100 \stackrel{\mathrm{N}}{\mathrm{N}}$ assays for the $\Delta \mathrm{F} 508$ defect ( 57 normal, $33 \mathrm{\omega}$ carriers, and 10 homozygous affected), all readings gave the expected 91 or 94 base pair $\stackrel{0}{C}$ fragment with the following narrow ranges. $\stackrel{\Phi}{D}$ Mutant allele: mean ( 1 SD), 91.35 (0.10 base pairs); coefficient of variation $(\mathrm{CV})=0.11 \%$. Wild-type allele: mean (1 SD), 94.39 (0.11 base pairs); $\mathrm{CV}=0.12 \%$.

\section{HUNTINGTON DISEASE}

To compare DNA fragment sizing in unstable triplet repeats, 79 cases referred for Huntington disease testing were studied in parallel, $\frac{\hat{\partial}}{\partial}$ using the conventional radioactive based assay $?$ and capillary. electrophoresis. The results from conventional assays were determined by visual inspection of autoradiographs by two independent observers. In one of the 79 cases, the diagnosis of Huntington disease would have 
Table 1 Comparison of $H D(C A G)_{n}$ triplet repeats determined by slab gel electrophoresis and capillary electrophoresis

\begin{tabular}{llll}
\hline & \multicolumn{2}{l}{ Results for two alleles } & \\
\cline { 2 - 4 } Example & Slab gel electrophoresis & Capillary electrophoresis & Comments \\
\hline 1 & $17 / 21$ & $15 / 19$ & Normal range \\
2 & $17 / 17$ & $15 / 15$ & Homozygous pattern \\
3 & $17 / 35-36$ & $15 / 35$ & Intermediate range \\
4 & $18 / 37-38$ & $16 / 36$ & Equivocal result \\
5 & $23 / 39$ & $21 / 38$ & Affected range \\
6 & $17 / 42-43$ & $15 / 42$ & Affected range \\
7 & $15 / 54$ & $13 / 52$ & High affected range \\
8 & $29 / 52$ & $26 / 26$ & False negative \\
9 & $17.69(3.66) /$ & $16.05(3.68) / 29.24(11.01)$ & Composite of 78 results \\
& $31.00(11.03)$ & & (excluding \#8) \\
\hline
\end{tabular}

Except for example 8, all 78 results obtained by capillary electrophoresis had an $\mathrm{n}$ value that was 1-2 repeats smaller than slab gel electrophoresis. Examples read differently by two scientists are reported as such. Corresponding capillary electrophoresis results were not subject to this variation. Example 9 represents the mean (1 SD) of 78 results and confirms the consistent 1-2 repeat difference between the two methods.

been missed by capillary electrophoresis because the mutant allele was not seen and the report, if issued, would have indicated a homozgyous normal pattern of $n=26$ for the (CAG) .

A discrepancy with capillary electrophoresis, similar to that described for cystic fibrosis, was also found when testing for the (CAG) $)_{n}$ repeat in Huntington disease. The current experience with quantitation of triplet repeats in a number of neurological disorders is based on slab gel electrophoresis. For example, a cut off used for the mutant allele in Huntington disease is an $\mathrm{n}$ value $>37$. However, this has to be redefined when the capillary electrophoresis results show consistently lower repeat numbers of between 1 and 2 fewer repeats (table 1). Like the cystic fibrosis results described earlier, the triplet repeat quantitation on capillary electrophoresis was highly reproducible. The different methods in use and the inherent problems of slab gels, when used to quantitate triplet repeats,
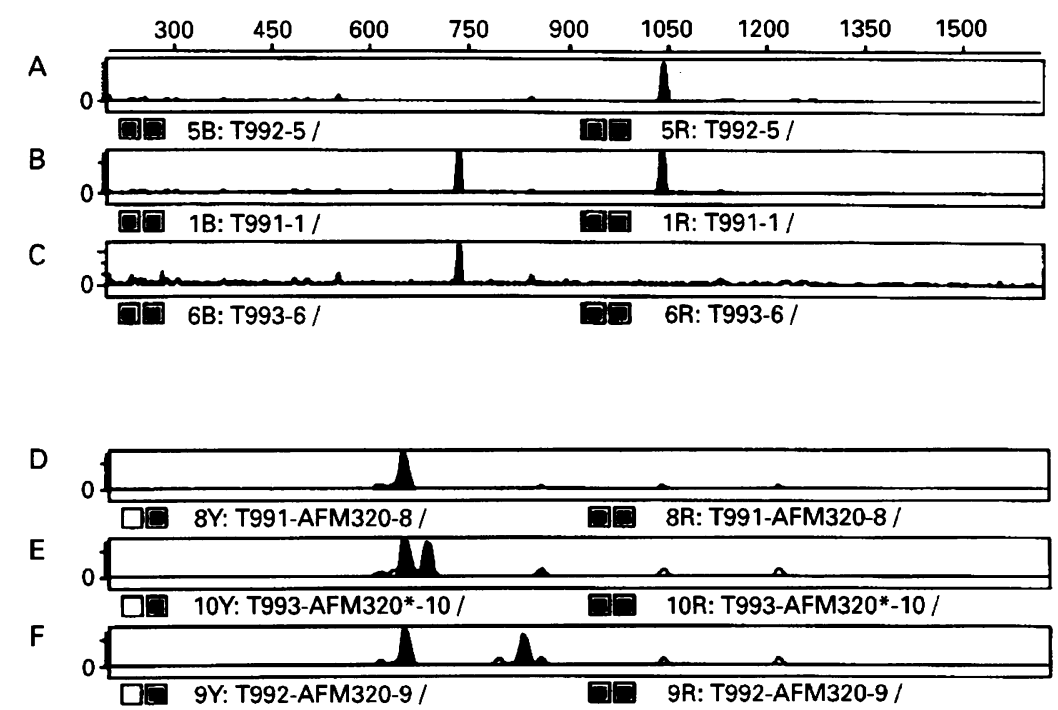

Figure 1 a Thalassaemia testing showing that the mother $(B)$ is heterozygous for the common SEA deletion and the fetus $(C)$ is homozygous for this defect because there is only the smaller (mutant) allele present. The father $(A)$ appears to be normal, which suggests non-paternity. Another explanation would be a second, more extensive deletion in the father and fetus which has not been detected by PCR. (D-F) Microsatellite analysis (AFM320) shows that the fetus (E) shares a common band with the mother $(D)$ and father $(F)$, but has a second band that is not found in his father; this result confirms non-paternity. Another feature of the traces illustrated is the co-migrating DNA size markers (small peaks at the bottom of each trace). This is possible because the size markers are labelled with a different fluorochrome to the PCR primers. has meant that $(\mathrm{CAG})_{\mathrm{n}}$ results are often qualified-for example, $37+/-2$ repeats. However, this is different from that experienced with capillary electrophoresis because in all 79 cases assayed, capillary electrophoresis generated results with a consistently lower $\mathrm{n}$ value than that obtained from slab gel electrophoresis (table 1).

$\alpha$ THALASSAEMIA

A five second injection time at $10 \mathrm{kV}$, followed by a 30 minute capillary electrophoresis run, enabled the mutant ( -697 base pairs) and wild-type alleles ( 1006 base pairs) to be separated adequately in a prenatal test for $\alpha$ thalassaemia resulting from a deletion of the South East Asian type (fig 1). Again, the sizing of the two alleles on capillary electrophoresis did not correspond to that expected from sequence data ( 660 and 980 base pairs) but, on this occasion, the difference was in the higher range. The discrepancy was attributed to the GS2500 TAMRA marker that was required because of the larger fragments present. This marker proved to be unsatisfactory, frequently giving split peaks, and making sizing particularly difficult because it was not clear which of the two peaks should be taken as the reference one. This artefact was not found with the smaller GS500 TAMRA molecular weight marker. In the above prenatal diagnosis example, the anomalous result depicted in fig 1 led to further testing using a chromosome 15 microsatellite (AFM320). This confirmed nonpaternity (fig 1).

\section{DNA SEQUENCING}

Three missense mutations (A149P, A174D, and $\mathrm{N} 334 \mathrm{~K}$ ) in the aldolase $B$ gene account for $\sim 70-90 \%$ of cases with hereditary fructose intolerance in the USA and UK. ${ }^{4}$ Two of these mutations (A149P, which is a $\mathrm{G}$ to $\mathrm{C}$ transversion, and $A 174 \mathrm{D}$, which is a $C$ to $A$ transversion) are found in exon 5 of the gene. In a family with this disorder, the proband was known to be heterozygous for the A149P mutation, which can be detected easily because it creates a new site for the restriction enzyme AcyI. However, the second mutation was unknown. To detect the latter, the relatively small exon 5 of the gene was sequenced rapidly by capillary electrophoresis. This showed the proband to be a compound heterozygote for the A149P and A174D defects.

Comparative studies of the ABI PRISM ${ }^{\mathrm{TM}}$ 310 Genetic Analyzer with the ABI PRISM ${ }^{\mathrm{TM}}$ 377 model, which uses fluorescent based technology with slab gel electrophoresis, gave the following results for DNA sequencing (table 2). These showed that for a relatively small sized amplicon, such as the aldolase $B$ gene, the ABI PRISMTM 310 Genetic Analyzer was very sensitive and gave both satisfactory and rapid results. However, for sequencing of a larger amplicon, such as would be required with the UBE3A gene (mutations in which give rise to the Angelman syndrome) ${ }^{5}$ the ABI PRISMTM 377 would be a more efficient option. 
Table 2 Comparison of direct sequencing of PCR derived products from the UBE3A and aldolase $B$ genes

\begin{tabular}{|c|c|c|}
\hline Feature & ABI PRISM ${ }^{\mathrm{TM}} 377$ & $\begin{array}{l}\text { ABI PRISM }{ }^{\mathrm{TM}} 310 \\
\text { Genetic Analyzer }\end{array}$ \\
\hline $\begin{array}{l}\text { Average length of sequence read } \\
\text { Number of samples processed in } 8 \text { hours } \\
\text { Running time per sample }\end{array}$ & $\begin{array}{l}600 \text { bases } \\
\text { Up to } 64 \\
\sim 7 \text { hours (irrespective of } \\
\text { template length) }\end{array}$ & $\begin{array}{l}350 \text { bases } \\
\text { Up to } 15 \\
\sim 30 \text { minutes (for } \\
\text { template of } 250 \mathrm{bp} \text { ) }\end{array}$ \\
\hline $\begin{array}{l}\text { Sensitivity } \\
\text { Injection volume } \\
\text { fmol of dye terminator/peak }\end{array}$ & $\begin{array}{l}-2 \mu \mathrm{l} \\
2 \mathrm{x} \text { fmol }\end{array}$ & $\begin{array}{l}\sim 0.1 \mu \mathrm{l} \\
0.016 \mathrm{x} \text { fmol }\end{array}$ \\
\hline
\end{tabular}

*Assume that at the completion of the reaction there are $\mathrm{x}$ pmol of sequencing product. For the ABI PRISM ${ }^{\mathrm{TM}} 377$ analysis, the product was dissolved in $4 \mu \mathrm{l}$ of buffer and for the ABI PRISM ${ }^{\mathrm{TM}}$ 310 it was dissolved in $24 \mu \mathrm{l}$ of buffer. A $2 \mu \mathrm{l}$ aliquot was used to load the ABI PRISM ${ }^{\mathrm{TM}} 377$ $(0.5 \mathrm{x} \mathrm{pmol})$ and an estimated $0.1 \mu \mathrm{l}$ aliquot was used for the ABI PRISM ${ }^{\mathrm{TM}} 310(0.004 \mathrm{x}$ pmol) For a reading of 250 bases in the aldolase $B$ gene sequence, each peak, on average, would represent $0.5 \times / 250$ pmol (ABI PRISM ${ }^{\mathrm{TM}} 377$ ) or $0.004 \times / 250 \mathrm{pmol}$ (ABI PRISM ${ }^{\mathrm{TM}} 310$ ) of dye terminator. Thus, the ABI PRISM ${ }^{\mathrm{TM}} 310$ is $\sim 10^{2}$ times more sensitive than the ABI PRISM ${ }^{\mathrm{TM}} 377$. The above calculation uses a conservative loading volume of $0.1 \mu \mathrm{l}$ for the capillary ${ }^{3}$ and is based on sequencing runs (ABI PRISMTM 377 and 310) that demonstrated comparable signal intensities.

costs

Over a period of approximately six months it was estimated that each sample electrophoresed with the ABI PRISM ${ }^{\text {TM }} 310$ Genetic Analyzer cost, in terms of consumables, an additional AUD $\$ 10$ to process. Although there are other automated means to undertake DNA analysis including sequencing (for example, the ABI PRISMTM 377), the ABI PRISMTM 310 was competitively priced with a platform cost of around AUD $\$ 80000$ compared with about AUD\$200 000 for the 377 model. There are cheaper instruments that allow capillary electrophoresis to be undertaken, but these do not have the multicolour capability.

\section{Discussion}

The numbers of forensic, industrial, and clinical applications that utilise capillary electrophoresis are growing. Analytes that can be separated by this technique are also extensive and include amino acids, proteins, drugs, chemicals, oligonucleotides, and DNA. ${ }^{2}$ In the area of DNA genetic diagnosis, capillary electrophoresis has been successful in detecting the G1691A mutation in the factor $\mathrm{V}$ gene, ${ }^{6}$ in mutation analysis for familial defective apolipoprotein B-100,7 and in screening of the p53 gene. $^{8}$

The present study describes the experience of a molecular genetics laboratory that provides a DNA diagnostic service for a range of genetic disorders. In this laboratory, the availability of capillary electrophoresis and, thus, the option to automate, has increased productivity by allowing staff additional time to use their skills in more relevant activities. Thus, it will become possible to increase the range of tests available, a move that would not have been considered when the manual methods were in use. Assays are now electrophoresed overnight and the results are scanned on the following day (each sample takes $\sim 30$ minutes to run). While a run is in progress, the ABI PRISM ${ }^{\mathrm{TM}} 310$ can be programmed to retest unsatisfactory samples or change the order of samples to be assayed.

The ability to use primers labelled with different coloured fluorochromes has expanded the potential for multiplexing, which is essential when dealing with genetic disorders produced by multiple mutations. Although there are other machines available for auto- mated DNA analysis, the comparable model to the ABI PRISMTM 310 (that is, one that offers multicolour fluorescence and both fragment analysis and sequencing capabilities) is the ABI PRISM ${ }^{\mathrm{TM}}$ 377. However, the latter is considerably more expensive to purchase. The impressive reproducibility demonstrated by the ABI PRISM ${ }^{\mathrm{TM}} 310$ Genetic Analyzer has improved the quality of the results coming from this laboratory because it takes away observer error in gel reading and the intrinsic deficiencies associated with slab gel electrophoresis. In association with this, the availability of hard data for comparative purposes, rather than estimations of DNA fragment sizes from slab is gels, will facilitate quality assurance.

One false negative result in the present study was obtained when testing for the Huntington disease mutation. However, it is more likely that this represented a PCR error, rather than a capillary electrophoresis problem, since the $O$ latter technique is more sensitive than slab gel or electrophoresis and two different PCRs were $\stackrel{\circ}{\rightarrow}$ involved. In cases in which a homozygous 은 result is obtained, reports are not issued until a second PCR using a different set of primers is undertaken. The additional work required to do this has been eased by developing a capillary electrophoresis based assay.

A problem with capillary electrophoresis is fragment sizing, which can differ from that. obtained with conventional slab gel electrophoresis. In some circumstances, such as forensic testing, these may not be significant because comparisons are being made. However, the detection of genetic disorders requires $\triangle$ both comparisons and, in the case of triplet $\overrightarrow{\bar{O}}$ repeat quantitation, the calculation of an absolute number. For Huntington disease, the $(\mathrm{CAG})_{\mathrm{n}}$ result will place an individual into one of three groups: normal, $\mathrm{n}<31$; intermediate, $\mathrm{n}=31-37$; and affected, $\mathrm{n}>37$. Interpreting results in this disorder becomes difficult when triplet repeats fall at the normal/intermediate 8 or intermediate/mutant cut off values. This could be resolved by: testing two populations 우 and determining the normal and affected $\supset$ triplet repeat ranges based on capillary electrophoresis, or providing DNA reports that place $N$ patients into the three groups by running the appropriate known controls with each assay. Either would be reasonable. The first option $\omega$ has the potential to cause confusion, since units would not be directly comparable unless it was

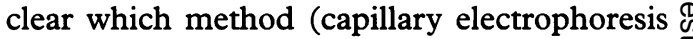
or slab gel) was followed. The second approach may also require identification of individuals who have an upper intermediate value for the $(\mathrm{CAG})_{\mathrm{n}}$ and, therefore, are at risk of transmit- $\frac{}{\mathbb{D}}$ ting a premutation or even Huntington disease to their offspring. ${ }^{9}$

The discrepancies in band sizing between slab gel electrophoresis and capillary electrophoresis can be explained partly by the different sieving effect of cross linked polyacrylamide compared to the linear polymer used in capillary electrophoresis. ${ }^{10}$ An electro-osmotic effect, which would alter the migration of DNA fragments, could be another factor. It is difficult to assess the latter variable, because 
the specific composition of the polymers is not obtainable from the manufacturers. However, the use of coated capillaries would exclude an electro-osmotic effect. ${ }^{3}$ We have discussed the sizing problem with the manufacturers on a number of occasions but, to date, no definitive explanations have been forthcoming.

As polymer technology is evolving rapidly, the range and types of separation matrices are considerable. $^{3}$ The changing of commercial polymers by the manufacturer (as happened to us) or the use of alternative polymers including "home made" ones will mean that mobility and, therefore, band sizes could change. The concentration of the amplicon used for capillary electrophoresis analysis can also be crucial because overloading has the potential to complicate data interpretation. If necessary, this can be avoided by checking the PCR products on minigels before capillary electrophoresis.

The routine diagnostic laboratory may be faced with mutation detection for a genetic disorder in which it would be easier to sequence a segment of DNA than test for individual mutations. This approach is illustrated by the hereditary fructose intolerance example in which two common mutations (A149P and A174D) are present in a small exon. As shown in this particular case, DNA sequencing allowed both to be detected or screened for in the one capillary electrophoresis run. On the other hand, capillary electrophoresis in its present form (a single capillary with samples loaded sequentially) is less suitable for sequencing large DNA fragments or for laboratories in which DNA sequencing is a major work component. This may change as the polymers for capillary electrophoresis are developed further-for example, a claim for the latest ABI polymer (Performance Optimized Polymer 6) is that it can resolve 600 bases in a shorter run time than would be possible with the 377 model.
The additional running costs associated with capillary electrophoresis need to be balanced by its usefulness. For a laboratory with a high throughput of DNA samples, the costs are reasonable because they are offset by the productivity gained. Each laboratory will need to assess its needs and resources in this respect. In view of the potential for this technology, laboratories that are involved in the provision of a DNA diagnostic service should consider utilising techniques that are more compatible with capillary electrophoresis. Thus, the value of hybridisation based strategies should be reviewed and more effort placed in those that rely on fragment size analysis.

This work was supported by the NHMRC and grants from the Rebecca L Cooper Medical Research Foundation and the Royal Prince Alfred Hospital Research Foundation. We thank Dr A Fimmel and Mr B Watson for their help with DNA analyses.

1 Cotton RGH. Slowly but surely towards better scanning for mutations. Trends Genet 1997;13:43-6.

2 Altria KD, ed. Capillary electrophoresis guidebook. PrinciAltria KD, ed. Capillary electrophoresis guidebook. Principles, operation and applications. In: Methods in mote
biology. Vol 52. New Jersey: Humana, 1996:1-122.

3 Barron AE, Blanch HW. DNA separations by slab gel and capillary electrophoresis: theory and practice. Sep Purif Method 1995;24:1-118.

4 Tolan DR, Brooks CC. Molecular analysis of common aldolase B alleles for hereditary fructose intolerance in North Americans. Biochem Med Metab Biol 1992;48:19-25.

5 Matsuura T, Sutcliffe JS, Fang P, Galjaard R-J, Jiang Y-H, Benton CS, et al. De novo truncating mutations in E6-AP ubiquitin-protein ligase gene (UBE3A) in Angelman ubiquitin-protein ligase gene (U

6 Van de Locht LTR, Kuypers AWHM, Verbruggen BW, Linssen PCM, Novakova IRO, Mensink EJBM. Semiautomated detection of the factor $\mathrm{V}$ mutation by allele specific amplification and capillary electrophoresis. Thromb Haemostas 1995;74:1276-9.

7 Lehmann R, Koch M, Pfohl M, Voelter W, Haring H-U, Liebich HM. Screening and identification of familial defective apolipoprotein B-100 in clinical samples by capillary gel electrophoresis. 7 Chromatogr 1996;744:187-94.

8 Katsuragi K, Kitagishi K, Chiba W, Ikeda S, Kinoshita M. Fluorescence-based polymerase chain reaction-singlestrand conformation analysis of p53 gene by capillary elecstrand conformation analysis of p53 gene by

9 Kremer B, Goldberg P, Andrew SE, Theilmann J, Telenius $\mathrm{H}$, Zeisler J, et al. A worldwide study of the Huntington's disease mutation. The sensitivity and specificity of measuring CAG repeats. $N$ Engl $\mathcal{F}$ Med 1994;330:1401-6.

10 Quesada MA. Replaceable polymers in DNA sequencing by capillary electrophoresis. Curr Opin Biotechnol 1997;8:8293. 